\title{
CAR-modified T cells targeting CD19—curing the incurable
}

Relapsed or refractory acute lymphoblastic leukaemia (ALL) is associated with an extremely poor prognosis and poses a considerable therapeutic challenge, especially for patients who do not achieve a second complete remission or who relapse after stem-cell transplantation. Engineered T-cell therapy is a new strategy for the treatment of this disease that has already demonstrated durable complete remissions in a small number of patients. One T-cell therapy approach uses chimeric antigen receptors (CAR). By infusing autologous $\mathrm{T}$ cells transduced with a CD19-targeting CAR (CTL019) lentiviral vector in 25 children and five adults with relapsed or refractory ALL, Stephan Grupp and coauthors reported a staggering $90 \%$ complete remission rate in 27 of these patients. Responses were seen in two patients who were refractory to the agent blinatumomab, an antibody that targets the CD19 antigen present on B cells.

Sustained remission was noted with an impressive 6-month event-free survival

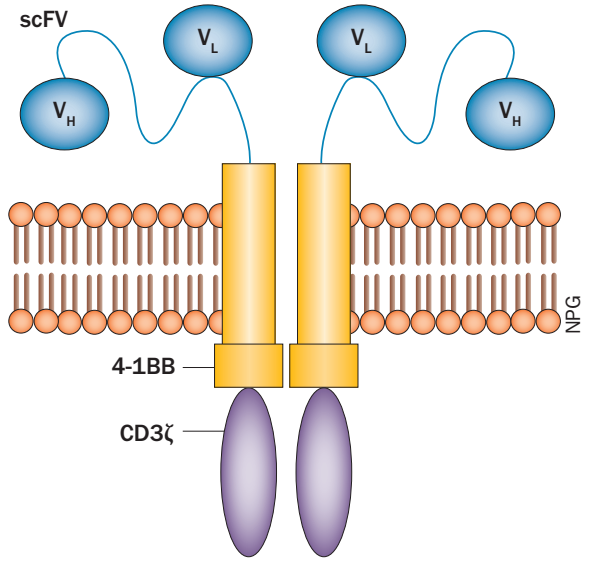

rate of $67 \%$ and an overall survival rate of $78 \%$ at 2 years. Of the 27 patients with complete remission, 19 remained in remission until study completion, and 15 received no further therapy. These results are considerably better than achieved with the most-recently approved chemotherapy drugs for relapsed ALL, which produce complete remission rates $<25 \%$, and median response durations of 4-9 weeks.
Severe cytokine-release syndrome-a systemic inflammatory response caused by elevated levels of cytokines-was the main adverse effect. All patients had at least mildto-moderate cytokine-release syndrome that required hospitalization, while eight patients developed severe cytokinerelease syndrome requiring intensive care and some degree of respiratory support; these responses were managed effectively with the IL- 6 blocking agent tocilizumab.

"A short term remission rate of $90 \%$ is far better than we could have expected when we started this study. We are particularly excited about the fact that we have survivors 1-2 years out who remain in remission without further therapy, such as bone marrow transplant," concludes Grupp.

\section{Lisa Hutchinson}

Original article Maude, S. L. et al. Chimeric antigen receptor T cells for sustained remissions in leukemia. N. Engl. J. Med. 371, 1507-1517 (2014) 